\title{
Avaliação do crescimento, do controle laboratorial e da corticoterapia em um grupo de pacientes com a forma clássica da deficiência da 21-hidroxilase
}

\author{
Evaluation of growth, laboratorial control and corticotherapy in a sample of patients \\ with the classical form of 21-hydroxylase deficiency
}

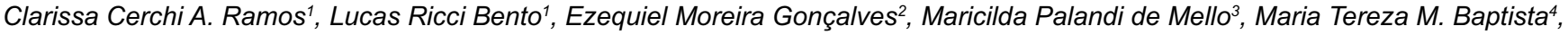

Sofia Helena V. de Lemos-Marini ${ }^{5}$, Gil Guerra-Júnior ${ }^{6}$
\end{abstract}

\section{RESUMO}

Objetivo: Avaliar o padrão de crescimento de pacientes com hiperplasia adrenal congênita com a forma clássica da deficiência da 21-hidroxilase (21-OH), em relação ao controle hormonal e ao uso de corticóide no tratamento.

Métodos: Análise retrospectiva dos prontuários de 45 pacientes. Como padrão de crescimento, foi utilizado o ganho ou não de altura, avaliando-se a diferença entre o escore $Z$ da estatura na última consulta (para idade óssea) em relação ao escore $Z$ da estatura no início do tratamento (para a idade cronológica). Foram avaliadas todas as concentrações de $17-\mathrm{OH}$ progesterona (17-OHP), androstenediona e renina, sendo considerados bem controlados os pacientes com $50 \%$ ou mais das dosagens normais. Em relação ao corticóide, foram analisados o tipo e a dose.

Resultados: A idade na última consulta variou de 2,8 a 26,6 anos (12,6 $\pm 5,8$ anos), sendo 31 do sexo feminino, 30 com a forma perdedora de sal; $62 \%$ foram considerados bem controlados para $17-\mathrm{OHP}, 75 \%$ para androstenediona e $78 \%$ para renina. A hidrocortisona foi usada por 41 pacientes $\left(20,2 \pm 2,6 \mathrm{mg} / \mathrm{m}^{2} / \mathrm{dia}\right)$ e, por 40 , em associação com a $9 \alpha$-fludrocortisona. Encontrou-se 14 pacientes com ganho, 20 com manutenção e 11 com perda estatural. Os pacientes perdedores de sal $(p=0,01)$ e os bem controlados $(p=0,0005)$ para $17-\mathrm{OHP}$ e androstenediona apresentaram associação significativa com o ganho de estatura.

Conclusões: Nesta amostra de pacientes com a forma clássica da deficiência da 21-OHD, o melhor crescimento apresentou associação com o bom controle laboratorial da 17-OHP e da androstenediona e com a forma perdedora de sal.

\footnotetext{
'Aluno de graduação da Faculdade de Ciências Médicas da Universidade Estadual de Campinas (FCM-Unicamp), Campinas, SP, Brasil

2Professor de Educação Física e mestrando em Saúde da Criança e do Adolescente pela FCM-Unicamp, Campinas, SP, Brasil

${ }^{3}$ Livre-docente em Genética Médica e pesquisadora do Centro de Biologia Molecular e Engenharia Genética da Unicamp, Campinas, SP, Brasil ${ }^{4}$ Professora doutora colaboradora do Departamento de Clínica Médica da FCM-Unicamp, Campinas, SP, Brasil

${ }^{5}$ Professora doutora do Departamento de Pediatria da FCM-Unicamp, Campinas, SP, Brasil

${ }^{6}$ Professor livre-docente do Departamento de Pediatria da FCM-Unicamp, Campinas, SP, Brasil
}

Palavras-chave: hiperplasia supra-renal congênita; esteróide 21-hidroxilase; hidrocortisona; progesterona; crescimento.

\section{ABSTRACT}

Objective: To verify the growth pattern of patients with congenital adrenal hyperplasia (CAH) due to classical 21hydroxylase $(21-\mathrm{OH})$ deficiency in relation to hormonal control and use of corticoid during the treatment.

Methods: Retrospective analysis of data from 45 patients. The growth pattern was analyzed according to height gain or not, using the difference between height $\mathrm{Z}$ score (for bone age) at the last visit in relation to the height $\mathrm{Z}$ score (for chronological age) in the first visit. Concentrations of 17-OH progesterone (17-OHP), androstenedione and rennin were evaluated, and the patients were considered well-controlled when $50 \%$ or more of the dosages were normal. Corticoid therapy was analyzed according to type and dose.

Results: The age at last visit ranged from 2.8 to 26.6 years (12.6 \pm 5.8 years), 31 were females, 30 with salt wasting form; $62 \%$ were considered well-controlled for $17-\mathrm{OHP}$, $75 \%$ for androstenedione and $78 \%$ for renin. Hidrocortisone was used in 41 patients $\left(20.2 \pm 2.6 \mathrm{mg} / \mathrm{m}^{2} /\right.$ day $)$ and 40 in association with $9 \alpha$-fludrocortisone. There were 14 patients with height gain, 20 with maintenance and 11 with loss. Height gain was associated with salt wasting patients $(p=0.01)$ and with patients well-controlled for 17OHP $(p=0,0005)$ and androstenedione $(p=0,02)$.

Fonte financiadora: CNPq, com bolsas para os alunos de iniciação científica e Fundação de Amparo à Pesquisa do Estado de São Paulo, com bolsa de mestrado, processo 06/01978-0

Endereço para correspondência

Gil Guerra-Júnior

Departamento de Pediatria da FCM-Unicamp

Cidade Universitária Zeferino Vaz, sem número Caixa Postal 6111

CEP 13083-970 - Campinas/SP

E-mail: gilguer@fcm.unicamp.br

Recebido em: 13/8/2007

Aprovado em: 5/10/2007 
Conclusions: In this sample of patients with CAH due to classical 21-OH deficiency, better height gain was associated with a good control of 17-OHP and androstenedione and with salt wasting clinical form of the disease.

Key-words: adrenal hyperplasia, congenital; steroid 21-hydroxylase; hydrocortisone; progesterone; growth.

\section{Introdução}

A hiperplasia adrenal congênita (HAC) é um grupo de doenças de herança autossômica recessiva, que se caracteriza por atividade reduzida ou ausente de uma das cinco enzimas envolvidas na síntese do cortisol, o que acarreta diminuição na concentração sérica de cortisol e aumento crônico do hormônio hipofisário adrenocorticotrófico (ACTH). Mais de 90\% dos casos de HAC ocorrem devido à deficiência da enzima 21-hidroxilase (21-OH) por mutações no gene CYP21, levando à diminuição de cortisol e aldosterona e aumento de 17-OH progesterona (17-OHP) e andrógenos ${ }^{(1)}$.

A HAC por deficiência 21-OH pode apresentar duas formas clínicas: a clássica, subdividida em perdedora de sal e virilizante simples; e a não clássica, subdividida em tardia e críptica. A forma clássica (HAC-C21-OHD), mais grave, caracteriza-se por virilização pré-natal no sexo feminino e virilização pós-natal em ambos os sexos, associadas ou não à perda de sal, que pode ocorrer independentemente do sexo $^{(1)}$.

A partir da suspeita clínica, o diagnóstico da forma clássica da doença é obtido pelas concentrações séricas basais muito elevadas de 17-OHP e androstenediona $(\Delta 4)$ e, nas formas perdedoras de sal, há hiponatremia, hipercalemia e atividade de renina plasmática aumentada ${ }^{(1)}$.

Diversos fatores parecem interferir na altura final de indivíduos com HAC-C21-OHD, como a idade ao diagnóstico e/ou início do tratamento, o controle laboratorial em longo prazo e a utilização por tempo prolongado de doses inadequadas de glicocorticóides em relação às necessidades habituais $^{(2-14)}$.

O tratamento com reposição de glicocorticóide tem por objetivo aumentar as concentrações séricas de cortisol, diminuindo as de ACTH e a elevação dos compostos prébloqueio enzimático ${ }^{(1)}$. Em face à sobrevida crescente dos afetados por HAC-C21-OHD com o uso de glicocorticóide, passou-se a buscar um melhor potencial de crescimento e uma altura final favorável ${ }^{(2-17)}$. O uso de mineralocorticóide, a $9 \alpha$-fludrocortisona, em pacientes com concentrações ele- vadas de renina plasmática, independentemente da presença do quadro clínico de perda de sal, é citado em alguns estudos como um fator para o crescimento normal ${ }^{(18,19)}$; contudo, outros estudos não demonstraram qualquer diferença na velocidade de crescimento ou no controle laboratorial em longo prazo, no que diz respeito àqueles sem evidência clínica de perda salina ${ }^{(20,21)}$.

Vários estudos mostraram que a supressão completa ou mesmo a normalização das concentrações séricas de 17-OHP e dos andrógenos não deve ser utilizada como objetivo do tratamento, pois pode indicar o uso excessivo de glicocorticóide, com conseqüente prejuízo na velocidade de crescimento ${ }^{(20-23)}$.

Portanto, diante desta lacuna na definição da interação crescimento, corticóide e andrógenos na HAC-C21-OHD, o objetivo deste estudo foi avaliar o padrão de crescimento de pacientes com HAC-C21-OHD em relação ao controle hormonal laboratorial e o tipo e dose de glicocorticóide utilizado ao longo do tratamento.

\section{Métodos}

No período de agosto de 2005 a maio de 2006, foi feito um estudo retrospectivo com análise dos prontuários dos pacientes vivos com diagnóstico clínico-laboratorial e molecular de HACC21-OHD em acompanhamento rotineiro no Ambulatório de Endocrinologia Pediátrica do Hospital de Clínicas da Universidade Estadual de Campinas (Unicamp), em Campinas, São Paulo ${ }^{(24-26)}$. O estudo foi realizado após autorização do responsável pelo paciente, com assinatura do termo de consentimento pós-informado aprovado pelo Comitê de Ética em Pesquisa da Faculdade de Ciências Médicas da Unicamp. O sigilo das informações foi preservado mediante a criação de um número para identificação dos pacientes, conservando sua privacidade, de acordo com as normas da Declaração de Helsinki.

Foram avaliados os seguintes dados em relação ao paciente: sexo; forma clínica da doença (virilizante simples ou perdedora de sal); idade de início do tratamento; concentrações séricas de 17-OHP, androstenediona e renina; idade óssea (avaliada pelo método Tanner \& Whitehouse 20 ossos - TW20); idade cronológica na última consulta do prontuário e dados de estatura e peso na consulta inicial, aos dois anos de idade, e na última.

A partir destes dados, foram calculados o tempo total de acompanhamento da doença (meses) e os escores de desvio padrão (escore $Z$ ) da estatura e do peso do paciente nas consultas determinadas, utilizando-se como referência os dados do National Center for Health Statistics (NCHS) de 2000. 
Foram também calculadas as seguintes variáveis: diferença entre os escores $Z$ da estatura na última consulta (para idades óssea e cronológica) em relação à inicial e aos dois anos de idade (para idade cronológica). Quanto a estas variáveis, os pacientes foram divididos em três grupos: $\geq+1$ DP (significando ganho estatural), entre $+0,9$ e $-0,9$ DP (significando manutenção estatural) e $\leq-1$ DP (significando perda estatural).

Para avaliar o grau de controle hormonal laboratorial, foram analisadas todas as concentrações séricas de 17-OHP, androstenediona e renina, comparando-as aos valores normais esperados para sexo, idade cronológica e desenvolvimento puberal. Os pacientes foram divididos segundo cada uma das dosagens em: bom controle, quando $50 \%$ ou mais das dosagens hormonais estavam de acordo com os valores normais esperados, e mau controle, quando menos de $50 \%$ das dosagens apresentavam valores normais.

Em relação ao tratamento, os pacientes foram divididos em grupos de acordo com o tipo de glicocorticóide (somente hidrocortisona até a última consulta ou outro glicocorticóide por pelo menos dois anos seguidos), com o uso ou não de mineralocorticóide e com a dose média do glicocorticóide utilizada, corrigida para hidrocortisona $(\geq 20$, entre 15 e 20 $\mathrm{e} \leq 15 \mathrm{mg} / \mathrm{m}^{2} /$ dia). A dose média foi calculada por meio da análise das doses descritas em cada consulta.

Tabela 1 - Distribuição do número de pacientes com deficiência da 21-hidroxilase em relação ao padrão de controle laboratorial da $17-\mathrm{OH}$ progesterona e da androstenediona $(\Delta 4)$ e em relação ao padrão de controle laboratorial de ambas as dosagens hormonais para as formas clínicas da doença

\begin{tabular}{lccccc}
\hline & & & & $\Delta \mathbf{4}$ \\
& & & + & & - \\
\hline \multirow{2}{*}{ 17-OHP } & & + & & 28 & 0 \\
& & - & 6 & & 11 \\
\hline \multicolumn{2}{c}{$\mathbf{1 7 - O H P}$} & & & $\mathbf{\Delta 4}$ & \\
& + & - & + & & - \\
\hline VS & 5 & 10 & 7 & 8 \\
PS & 23 & 7 & 27 & 3 \\
\hline
\end{tabular}

+=bom controle; -=mau controle; $\mathrm{VS}=$ =virilizante simples; PS=perdedora de sal
Inicialmente, foi realizada análise descritiva dos dados e, depois, os dados foram analisados por qui-quadrado ou teste exato de Fisher e teste não paramétrico de Wilcoxon, com nível de significância de 0,05 .

\section{Resultados}

Entre os 45 pacientes com HAC-C21-OHD analisados, 31 (69\%) eram do sexo feminino e 30 (67\%) eram perdedores de sal. Não foi observada diferença na distribuição da forma clínica em relação ao sexo $(p=0,74)$. A idade de início do acompanhamento no serviço foi $2,4 \pm 3,7$ anos ( 0 a 13,9, com mediana de 0,3 anos). A idade na última consulta existente no prontuário foi $12,6 \pm 5,8$ anos (2,8 a 26,6, com mediana de 12,7 anos). Assim, o tempo total de acompanhamento foi $10,2 \pm 5,3$ anos (1,9 a 21,5, com mediana de 9,8 anos). A idade óssea avaliada na última consulta foi $12,5 \pm 5,3$ anos (2 a 18, com mediana de 14,5 anos). Quando avaliada a última consulta existente no prontuário, não foi observada diferença significante entre as idades cronológica e óssea, o mesmo ocorrendo quando separados apenas os casos com a forma virilizante simples ou perdedora de sal. Aos dois anos de idade, apenas 32 pacientes estavam em acompanhamento no serviço e a idade foi de $2,1 \pm 0,3$ anos (1,3 a 2,9, com mediana de 2,1 anos).

$\mathrm{Na}$ análise do controle laboratorial, 28 pacientes (62\%) apresentaram bom controle da 17-OHP, $34(75 \%)$ da androstenediona e 35 (78\%) da renina. Em um paciente, a renina nunca foi dosada. Foi observada associação significante entre os valores de 17-OHP e androstenediona (Fisher; $p<0,001$ ), conforme Tabela 1. O mesmo não ocorreu em relação à 17-OHP e renina e entre androstenediona e renina. Quando o controle laboratorial foi avaliado segundo a forma da doença, observou-se que os perdedores de sal controlaram mais a 17-OHP (qui-quadrado; $p=0,001$ ) e androstenediona (Fisher; $p=0,03$ ), como demonstra a Tabela 1 , do que os portadores da forma virilizante simples. $\mathrm{O}$ mesmo não ocorreu em relação à renina.

A Tabela 2 mostra os dados do escore $Z$ do peso e da estatura na consulta inicial, aos dois anos de idade e na última consulta existente no prontuário. Foi observada melhora significativa

Tabela 2 - Dados de escore Z do peso e da estatura nas consultas dos 45 pacientes com a forma clássica da deficiência da 21-OH

\begin{tabular}{lcccccc}
\hline Consulta & Primeira & $\begin{array}{c}\text { Z peso } \\
\text { Dois anos }\end{array}$ & Última & Primeira & $\begin{array}{c}\text { Z estatura } \\
\text { Dois anos }\end{array}$ & Última \\
\hline $\mathrm{n}$ & 45 & 32 & 45 & 45 & 32 & 45 \\
Média & $-1,1$ & $-0,4$ & 0,4 & $-0,8$ & $-0,9$ & $-0,6$ \\
Mediana & $-1,3$ & $-0,4$ & 0,1 & $-1,2$ & $-0,8$ & $-0,5$ \\
DP & 1,7 & 1,3 & 1,4 & 2,0 & 0,9 & 1,2 \\
Mínimo & $-4,2$ & $-4,1$ & $-3,0$ & $-5,8$ & $-3,1$ & $-3,9$ \\
Máximo & 2,3 & 2,8 & 4,3 & 4,0 & 1,3 & 2,2 \\
\hline
\end{tabular}


quando comparado o escore $Z$ do peso aos dois anos em relação ao inicial (Wilcoxon; $p<0,001$ ), da última consulta em relação à inicial (Wilcoxon; $p<0,001$ ) e da última consulta em relação aos dois anos (Wilcoxon; $p<0,001$ ). O mesmo ocorreu em relação ao escore $Z$ da estatura aos dois anos para a inicial (Wilcoxon; $p=0,01$ ) e ao da última consulta para os dois anos (Wilcoxon; $p=0,006$ ); porém, não foi observada diferença significativa do escore $Z$ da estatura na última consulta em relação à inicial.

Esta mesma análise, quando separados os casos por forma clínica, mostrou resultados diferentes. Entre os perdedores de sal, para o escore $Z$ do peso, houve melhora significativa entre a consulta aos dois anos em relação à inicial (Wilcoxon; $p<0,001$ ); entre a última consulta em relação à inicial (Wilcoxon; $p<0,001$ ) e entre a última consulta em relação aos dois anos (Wilcoxon; $p<0,001$ ). Ainda para os perdedores de sal, em relação ao escore $Z$ da estatura, observou-se melhora significativa entre a consulta aos dois anos em relação à inicial (Wilcoxon; $p=0,01$ ), entre a última consulta em relação à inicial (Wilcoxon; $p=0,008$ ) e entre a última consulta em relação aos dois anos (Wilcoxon; $p=0,002$ ). Para os portadores da forma virilizante simples, não foi observada diferença significativa tanto para o escore $Z$ do peso como para o da estatura, em nenhuma das três situações comparativas avaliadas.

Tabela 3 - Distribuição da 17-OH progesterona, da androstenediona $(\Delta 4)$, da renina e das formas clínicas da deficiência da $21-\mathrm{OH}$ em relação à diferença do escore $Z$ da estatura na última para a primeira consulta

\begin{tabular}{lcccc}
\hline & & \multicolumn{3}{c}{ ZE (IO) última - ZE inicial } \\
& & Ganho & Manutenção & Perda \\
\hline \multirow{2}{*}{ 17-OHP } & + & 14 & 11 & 3 \\
\multirow{4}{*}{4} & - & 0 & 9 & 8 \\
\multirow{4}{*}{ Renina } & + & 14 & 14 & 6 \\
\multirow{2}{*}{ Doença } & - & 0 & 6 & 5 \\
& + & 10 & 15 & 10 \\
& PS & 4 & 4 & 1 \\
& VS & 13 & 13 & 4 \\
\hline
\end{tabular}

$Z E=$ escore $Z$ da estatura; $1 \mathrm{O}=$ idade óssea; +=bom controle -=mau controle
Quando foi feita a análise da diferença do escore $Z$ da estatura (para a idade cronológica) na última consulta existente no prontuário em relação à inicial, encontrou-se $0,2 \pm 2,0$ (-6,1 a 5,3; mediana 0,3), sendo que em dez casos houve piora, em 20 manutenção e em 15 melhora. Fazendo a mesma análise, porém, utilizando o escore $Z$ da estatura em relação à idade óssea na última consulta, encontrou-se $-0,1 \pm 0,1$ (-6,2 a 8,4; mediana 2,6), sendo que em 14 casos houve melhora, em 20 manutenção e em 11 piora estatural.

$A$ análise da diferença do escore $Z$ da estatura (para a idade cronológica) na última consulta existente no prontuário em relação aos dois anos revelou valor de $0,4 \pm 0,9$ (-2,4 a 2,1; mediana 0,5$)$, com piora em três casos, manutenção em 23 e melhora em seis. A mesma análise para o escore $Z$ da estatura em relação à idade óssea na última consulta mostrou valor de $0,4 \pm 1,8(-2,4$ a 7,0; mediana 0,2$)$, notando-se piora em seis casos, manutenção em 20 e melhora em seis.

A Tabela 3 mostra a associação entre o controle hormonal da 17-OHP, da androstenediona e da renina e a forma clínica da doença, respectivamente, em relação à diferença do escore $\mathrm{Z}$ da estatura na última consulta (idade óssea) para a inicial. Foi observada associação entre o controle da 17-OHP e a melhora da altura na última consulta (qui-quadrado; $p<0,001$ ), o mesmo ocorrendo com a androstenediona (qui-quadrado; $p=0,02)$, não sendo observada, contudo, esta associação com a renina. Também foi observada associação entre a forma clínica da doença e a melhora da altura na última consulta entre os perdedores de sal (qui-quadrado; $p=0,01$ ).

Em relação ao tratamento, $41(90 \%)$ usaram na maior parte do tratamento hidrocortisona, quatro prednisona e 40 (88\%) receberam mineralocorticóide. A dose de glicorticóide, corrigida para hidrocortisona, utilizada durante todo o acompanhamento, foi $20,2 \pm 2,6 \mathrm{mg} / \mathrm{m}^{2} / \mathrm{dia}$ (12 a 26 ; mediana 20,2 ) e a utilizada nos primeiros dois anos de vida foi $22,8 \pm 3,3 \mathrm{mg} /$ $\mathrm{m}^{2} /$ dia (19 a 37; mediana 23). A dose utilizada nos primeiros dois anos de vida foi significativamente maior que durante todo o acompanhamento (Wilcoxon; $p<0,001$ ).

Tabela 4 - Distribuição da dose de glicocorticóide (hidrocortisona equivalente) durante todo o tratamento e apenas nos dois primeiros anos de vida em relação à diferença do escore $Z$ da estatura na última para a primeira consulta, em pacientes com a forma clássica da deficiência da $21-\mathrm{OH}$

\begin{tabular}{lcccc}
\hline & & Ganho & $\begin{array}{c}\text { ZE (IO) última - ZE inicial } \\
\text { Manutenção }\end{array}$ & Perda \\
\hline Glicocorticóide total & $\leq 15$ & 0 & 0 & 1 \\
(mg/m²/dia) & $15,1-19,9$ & 5 & 7 & 6 \\
Glicocorticóide dois anos & $\geq 20$ & 14 & 20 & 11 \\
(mg/m²/dia) & $15,1-19,9$ & 2 & 1 & 1 \\
\hline
\end{tabular}

$Z E=$ escore $Z$ da estatura; $I O=$ =idade óssea 
A Tabela 4 mostra a associação entre a dose de glicocorticóide durante todo o tratamento e nos dois primeiros anos de vida, respectivamente em relação à diferença de escore $Z$ da estatura na última consulta (idade óssea) para a inicial. Não foi observada associação entre a dose de glicocorticóide durante todo o tratamento e nos dois primeiros anos de vida em relação à melhora da altura na última consulta.

\section{Discussão}

Por ser uma doença autossômica recessiva, a HAC-C21OHD deveria apresentar distribuição semelhante de indivíduos afetados de ambos os sexos. No entanto, tanto no presente estudo como em outros da literatura, há predomínio do sexo feminino, provavelmente devido aos óbitos do sexo masculino sem diagnóstico na forma perdedora de sal. A distribuição de $2 / 3$ dos casos na forma perdedora de sal também é compatível com o que é descrito na literatura ${ }^{(1)}$.

A idade de início de acompanhamento no serviço foi variável, devido a diversos fatores. O diagnóstico da forma perdedora de sal é freqüentemente realizado nas primeiras semanas de vida, enquanto que o da forma virilizante simples é, em geral, mais tardio, especialmente no sexo masculino, quando aparecem os sinais de virilização com pseudopuberdade precoce. Vale lembrar que alguns casos iniciaram o acompanhamento no serviço depois de diagnosticados e tratados por algum tempo em outro serviço, e, também, irmãos dos casos índices, tanto na forma perdedora de sal como virilizante simples, foram diagnosticados intra-útero ou logo após o nascimento. No entanto, é importante salientar que o tempo de acompanhamento dos pacientes do presente estudo foi relativamente longo, com média de dez anos, o que, associado ao número expressivo de casos de uma doença relativamente rara, subsidiam os resultados encontrados.

O fato de se ter, na última consulta avaliada, uma idade cronológica média de 12,5 anos e mediana de 14,5 anos talvez possa ser a justificativa de não ter sido encontrada diferença estatística significativa entre as idades óssea e cronológica nesta avaliação.

No presente estudo, na análise das dosagens hormonais de 17-OHP, androstenediona e renina, observou-se porcentagem expressiva de pacientes que apresentaram mais de $50 \%$ destas dosagens com valores normais para a idade, sexo e desenvolvimento puberal. Para alguns autores, isto não necessariamente significa um controle bom, podendo inclusive indicar o uso de doses excessivas de corticóides ${ }^{(18,20,27,28)}$. Silva et al $^{(20)}$ observaram velocidade de crescimento normal mesmo com 17-OHP excedendo sete vezes os valores de referência e, quando buscaram normalizar a 17-OHP, houve diminuição significativa da velocidade de crescimento. Outros pesquisadores consideram o controle laboratorial um dos objetivos a ser alcançado no tratamento da HAC-C21-OHD, sendo que a maioria orienta para que se faça um controle efetivo da androstenediona e não se espere a supressão completa da 17-OHP, o que foi observado na maioria dos casos deste estudo considerados bem controlados ${ }^{(5,23,29,30)}$.

$\mathrm{O}$ fato de os pacientes com a forma perdedora de sal apresentarem melhor controle laboratorial do que os portadores da forma virilizante simples pode ter várias explicações, como o início precoce do tratamento, a maior aderência ao tratamento pelo conhecimento do risco de morte e a associação de mineralocorticóides na terapêutica, entre outras.

É importante e interessante salientar a melhora observada apenas entre os portadores da forma perdedora de sal, tanto na avaliação do peso como da estatura, aos dois anos e na última consulta em relação à inicial, o que pode ser interpretado como maior aderência ao tratamento devido à gravidade maior desta variante da doença. Quando não se avalia a magnitude do ganho ou da perda de peso e estatura e se analisa apenas numericamente quantos ganharam ou perderam estatura, os pacientes com a forma perdedora de sal também apresentaram associação positiva com o ganho estatural.

No presente estudo, a avaliação de quantos ganharam estatura foi feita comparando-se o escore $Z$ da estatura para a idade óssea na última consulta em relação ao escore $\mathrm{Z}$ da estatura para a idade cronológica inicial, o que mostrou equilíbrio entre os que ganharam (14 casos) e os que perderam estatura (11 casos), sendo que os outros 20 casos mantiveram a altura.

Diferentemente da maioria dos estudos da literatu$\mathrm{ra}^{(5,8,15,18,27)}$, observou-se associação entre os que ganharam estatura final e o controle laboratorial da 17-OHP. Também foi observada associação entre a melhora da altura final e o controle laboratorial da androstenediona, como notado por alguns autores ${ }^{(27,29,30)}$. Geralmente, o controle da 17-OHP está associado às doses excessivas de glicocorticóides, no entanto, parece que o acompanhamento intensivo e a orientação permanente das famílias permitem maior aderência ao tratamento e melhor controle clínico e laboratorial. Não foi possível, nesta investigação, avaliar a associação entre o ganho na altura final e a época de início do tratamento, como destacado por vários autores ${ }^{(5,6,9,10,12,15)}$ - pois em vários casos aqui analisados, o início do tratamento não foi realizado no serviço. 
O tratamento convencional de reposição com glicocorticóides na HAC-C21-OHD possui dois extremos que devem ser evitados. Um deles é o não tratamento ou o uso de baixas doses, com conseqüente estado de hiperandrogenismo responsável pela aceleração da maturação esquelética e fusão prematura das cartilagens de crescimento ${ }^{(5,15,16,31)}$. Por outro lado, o uso de doses suprafisiológicas, quer durante a manutenção, quer durante as intercorrências clínicas, especialmente nos primeiros dois anos de vida, resulta em hipercortisolismo iatrogênico, que também compromete o crescimento e a altura final ${ }^{(3,5,12,16,31)}$. Os diversos esquemas terapêuticos utilizados no tratamento da HAC-C21-OHD não diferem em relação ao grau de supressão do eixo hipotálamo-hipofisário, porém, o mesmo não ocorre quanto aos efeitos colaterais, em especial sobre o crescimento estatural ${ }^{(17)}$. O tipo, a dose e o modo de administração do glicocorticóide, além de sua associação a mineralocorticóides, devem ser considerados na escolha de um tratamento de reposição em longo prazo, na infância e na adolescência ${ }^{(1)}$.

\section{Referências bibliográficas}

1. White PC, Speiser PW. Congenital adrenal hyperplasia due to 21-hydroxylase deficiency. Endocr Rev 2000;21:245-91.

2. Gussinyé M, Potau N, Vicens-Calvet E, Albisu MA, Yeste D, Ibañez L et al. Talla adulta, patrón de crecimento y desarrollo puberal en pacientes con hiperplasia suprarrenal congénita, forma perdedora de sal. Med Clin (Barc) 1997;108:87-90.

3. Ercan O, Hatemi S, Kutlu E, Turan N. Effect of treatment on growth in congenital adrenal hyperplasia. Indian J Pediatr 2000;67:783-9.

4. Cabrera MS, Vogiatzi MG, New MI. Long term outcome in adult males with classic congenital adrenal hyperplasia. J Clin Endocrinol Metab 2001;86:3070-8.

5. Eugster EA, Dimeglio LA, Wright JC, Freidenberg GR, Seshadri R, Pescovitz $\mathrm{OH}$. Height outcome in congenital adrenal hyperplasia caused by 21-hydroxylase deficiency: a meta-analysis. J Pediatr 2001;138:26-32.

6. Schwartz RP. Back to basics: early diagnosis and compliance improve final height outcome in congenital adrenal hyperplasia. J Pediatr 2001; 138:3-5.

7. Freire POA, Guerra-Júnior G. Hiperplasia congênita das supra-renais por deficiência clássica de 21-hidroxilase: atualização com enfoque para a estatura final. Rev Paul Pediatr 2001;19:177-82.

8. New Ml; International Workshop on Management of Puberty for Optimum Auxological Results. Factors determining final height in congenital adrenal hyperplasia. J Pediatr Endocrinol Metab 2001;14 (Suppl 2):S933-7.

9. Hargitai G, Sólyom J, Battelino T, Lebl J, Pribilincová Z, Hauspie R et al. Growth patterns and final height in congenital adrenal hyperplasia due to classical 21-hydroxylase deficiency. Results of a multicenter study. Horm Res 2001;55:161-71.

10. Van der Kamp HJ, Otten BJ, Buitenweg N, De Muinck Keiser-Schrama SM, Oostdijk W, Jansen $\mathrm{M}$ et al. Longitudinal analysis of growth and puberty in 21-hydroxylase deficiency patients. Arch Dis Child 2002;87:139-44.
A dose de glicocorticóide, freqüentemente hidrocortisona administrada três vezes ao dia, foi a habitualmente recomendada para a reposição (15 a $20 \mathrm{mg} / \mathrm{m}^{2} /$ dia), com doses médias necessariamente maiores nos dois primeiros anos de vida, especialmente entre os perdedores de sal nas situações de estresse. Como as doses médias não apresentaram grandes variações e como a maioria dos pacientes utilizou mineralocorticóide, não foi observada associação entre a dose do glicocorticóide e a associação do mineralocorticóide com o ganho ou não de estatura na última consulta.

Portanto, o presente estudo, apesar das limitações de um estudo retrospectivo, permitiu concluir que, nesta amostra de 45 pacientes com HAC-C21-OHD, o melhor crescimento apresentou associação apenas com o bom controle laboratorial da 17-OHP e com a forma perdedora de sal.

\section{Agradecimentos}

Ao $\mathrm{CNPq}$, pelas bolsas de iniciação científica (LRB e CCAR), e à Fapesp, pela bolsa de mestrado (EMG).

11. Manoli I, Kanaka-Gantenbein Ch, Voutetakis A, Maniati-Christidi M, DacouVoutetakis C. Early growth, pubertal development, body mass index and final height of patients with congenital adrenal hyperplasia: factors influencing outcome. Clin Endocrinol (Oxf) 2002;57:669-76.

12. Brunelli VL, Russo G, Bertelloni S, Gargantini L, Balducci R, Chiesa L et al. Final height in congenital adrenal hyperplasia due to 21-hydroxylase deficiency: the Italian experience. J Pediatr Endocrinol Metab 2003;16 (Suppl 2):S277-83.

13. Lemos-Marini SH, Guerra-Júnior G, Morcillo AM, Baptista MT, Silva LO, Maciel-Guerra AT. Hiperplasia congênita das supra-renais por deficiência da 21-hidroxilase: altura final de 27 pacientes com a forma clássica. Arq Bras Endocrinol Metabol 2005;49:902-7.

14. Nguyen AT, Brown JJ, Warne GL. Growth in congenital adrenal hyperplasia Indian J Pediatr 2006;73:89-93.

15. Jääskeläinen J, Voutilainen R. Growth of patients with 21-hydroxylase deficiency: an analysis of the factors influencing adult height. Pediatr Res 1997;41:30-3.

16. Klingensmith GJ, Garcia SC, Jones HW, Migeon CJ, Blizzard RM. Glucocorticoid treatment of girls with congenital adrenal hyperplasia: effects on height, sexual maturation, and fertility. J Pediatr 1977;90:996-1004.

17. Winterer J, Chrousos GP, Loriaux DL, Cutler GB Jr. Effect of hydrocortisone dose schedule on adrenal steroid secretion in congenital adrenal hyperplasia. J Pediatr 1985;106:137-42.

18. Young MC, Ribeiro J, Hughes IA. Growth and body proportions in congenital adrenal hyperplasia. Arch Dis Child 1989;64:1554-8.

19. Yu ACM, Grant DB. Adult height in women with early-treated congenital adrenal hyperplasia (21-hydroxylase type): relation to body mass index in earlier childhood. Acta Paediatr 1995;84:899-903.

20. Silva IN, Kater CE, Cunha CF, Viana MB. Randomized controlled trial growth effect of hydrocortisone in congenital adrenal hyperplasia. Arch Dis Child 1997;77: 214-8 
21. Silva IN, Cunha CF, Antonio SD, Andrade GF. Ritmo de crescimento de crianças com hiperplasia congênita da supra-renal em tratamento com baixas doses de hidrocortisona. Arq Bras Endocrinol Metabol 2005;49:120-5.

22. Young MC, Hughes IA. Response to treatment of congenital adrenal hyperplasia in infancy. Arch Dis Child 1990;65:441-4

23. Cunha HM, Elias LL, Camacho-Hübner C, Moreira AC, Martinelli CE Jr. Different states of clinical control are associated with changes in IGF-I and IGFBPs in children with congenital adrenal hyperplasia due to 21-hydroxylase deficiency. Clin Endocrinol (Oxf) 2004;61:94-101.

24. de-Araújo M, Sanches MP, Suzuki LA, Guerra G, Jr Farah SB, de-Mello MP. Molecular analysis of CYP21 and C4 genes in Brazilian families with the classical forms of the steroid 21-hydroxylase deficiency. Braz J Med Biol Res 1996;29:1-13

25. Paulino LC, Araújo M, Guerra G Jr, Marini SH, De Mello MP. Mutation distribution and CYP21/C4 locus variability in Brazilian families with the classical form of the 21-hydroxylase deficiency. Acta Paediatr 1999;88:275-83.
26. Lau IF, Soardi FC, Lemos-Marini SH, Guerra-Júnior G, Baptista MTM, De Mello MP. H28+C insertion in the CYP21 gene: a novel frameshift mutation in a Brazilian patient with the classical form of 21-hydroxylase deficiency. J Clin Endocrinol Metab 2001;86:5877-80.

27. DiMartino-Nardi J, Stoner E, O'Connell A, New MI. The effect of treatment of final height in classical congenital hyperplasia. Acta Endocrinol (Copenh) 1986;279:305-14.

28. Brook CG, Zachmann M, Prader A, Murset G. Experience with long-term therapy in congenital adrenal hyperplasia. J Pediatr 1974;85:12-9.

29. Speiser PW. Congenital adrenal hyperplasia owing to 21-hydroxylase deficiency. Endocrinol Metab Clin North Am 2001;30:31-59.

30. Migeon CJ, Wisniewski AB. Congenital adrenal hyperplasia owing to 21hydroxylase deficiency. Growth, development and therapeutic considerations. Endocrinol Metab Clin North Am 2001;30:193-206.

31. Balsamo A, Cicognani A, Baldazzi L, Barbaro M, Baronio F, Gennari M et al. CYP21 genotype, adult height, and pubertal development in 55 patients treated for 21-hydroxylase deficiency. J Clin Endocrinol Metab 2003;88:5680-8. 